\title{
Clino-Pathological Features of Urinary Tract Infection in Rural India
}

\author{
Rutuja Raval1, R. J. Verma1, Hiten Kareliya ${ }^{2,3}$ \\ ${ }^{1}$ Department of Zoology, Gujarat University, Ahmedabad, India \\ ${ }^{2}$ Global Baroda Hospital, Vadodara, India \\ ${ }^{3}$ Narhari Hospital, Vadodara, India \\ Email: rutujaraval@gmail.com
}

Received 21 August 2015; accepted 18 October 2015; published 21 October 2015

Copyright (C) 2015 by authors and Scientific Research Publishing Inc.

This work is licensed under the Creative Commons Attribution International License (CC BY).

http://creativecommons.org/licenses/by/4.0/

(c) (i) Open Access

\section{Abstract}

The study was aimed to understand the clino-pathological characteristics of urinary tract infection along with the techniques used in diagnosis and treatment of the presenting infection. The study takes into consideration the various risk factors such as age, sex, and diabetes mellitus which can precipitate a urinary tract infection. The study was conducted at the Global Baroda Hospital, Vadodara and Narhari Hospital, Vadodara in the duration from January to March 2012, under the supervision of Dr. Hiten Kareliya. A questionnaire was prepared in accordance to evaluate risk factors of urinary tract infection. The patients under study were chosen according to specific inclusion criteria. The uropathogens were isolated with the help of biochemical testing. E. coli (38\%) was found to be the most prevalent organism followed by Klebsiella and Candida albicans (both $10 \%)$, Pseudomonas aeruginosa (9\%), Staphylococcus (7\%).

\section{Keywords}

Urinary Tract Infections, Bacteremia, Uropathogens, E. coli, Antibiotic Sensitivity, Aminoglycoside, Carbapenem, Catheterization, Diabetes, Calculi, Urosepsis

\section{Introduction}

Urinary tract infection (UTI) is defined as the microbial invasion of any of the tissues of the urinary tract extending from the renal cortex to the urethral orifice. Usually classified by the site of infection (the bladder [cystitis], kidney [pyelonephritis], or urine [bacteriuria]), UTI can be asymptomatic or symptomatic, characterized by a wide spectrum of symptoms ranging from mild irritative voiding to bacteremia. Infections that occur in a normal urinary tract with no prior instrumentation are considered “uncomplicated," whereas “complicated” in- 
fections are diagnosed in urinary tracts that have structural or functional abnormalities, including instrumentation such as indwelling urethral catheters, and are frequently asymptomatic [1]. The symptoms of a UTI include pain or a burning sensation during urination (dysuria), a frequent need to urinate, and lower abdominal pain.

Normally, urine is sterile. It is usually free of bacteria, viruses and fungi, but does contain fluids, salts and waste products. Any abnormality of the urinary tract that obstructs the flow of urine (a kidney stone, for example) sets the stage for an infection. An enlarged prostate gland also can slow the flow of urine, thus raising the risk of infection. Diabetics have a higher risk of acquiring a UTI because of changes in the immune system. Any other disorder that suppresses the immune system raises the risk of a urinary infection [2]. The common uropathogens identified in patients with UTI include enteric gram-negative bacteria, with $E$. coli being the most common followed by Klebsiella, Pseudomonas aeruginosa, and Proteus mirabilis [3].

Asymptomatic bacteriuria (ASB) is defined as the isolation of bacteria from the urine in significant quantities consistent with infection, but without the local or systemic urinary signs or symptoms [4]. Complications of an UTI are uncommon, but malefic when present, leading to kidney failure, where the kidneys lose almost all of their functioning capability, resulting in poisoning of the blood, also known as urosepsis [5].

Among males, UTIs occur with substantial incidence only at the extremes of life. One percent of boys may have a symptomatic UTI, most presenting within their first month. Whether the presence of a prepuce warrants a diagnosis of a complicated urinary tract is definitional; circumcised infants are at 10 to 20 times more at risk of contracting UTI. After infancy, the incidence of UTI among males is low, but infections do occur and some episodes may be complicated by prostatitis. In older men, symptomatic infection and asymptomatic bacteriuria are often complicated UTIs, usually attributable to benign prostatic hypertrophy [6].

It is observed in India that UTI accounts for nearly 7 million office visits and 1 million emergency department visits, resulting in about 100,000 hospitalizations [7]. Nevertheless, it is difficult to accurately assess the incidence of UTIs, because they are not reportable diseases in India as well as many other countries such as the United States. This situation is further complicated by the fact that accurate diagnosis depends on both the presence of symptoms and a positive urine culture, although in most outpatient settings this diagnosis is made without the benefit of culture. Women are significantly more likely to experience UTI than men. Nearly 1 in 3 women will have had at least 1 episode of UTI requiring antimicrobial therapy by the age of 24 years. Almost half of all women will experience 1 UTI during their lifetime [8].

Specific subpopulations at increased risk of UTI include infants, pregnant women, the elderly, patients with spinal cord injuries and/or catheters, patients with diabetes or multiple sclerosis, patients with acquired immunodeficiency disease syndrome/human immunodeficiency virus, and patients with underlying urologic abnormalities [9] [10]. Catheter-associated UTI is the most common nosocomial infection, accounting for $>1$ million cases in hospitals and nursing homes [11].

Micro-organisms can reach the urinary tract by haematogenous or lymphatic spread, but there is abundant clinical and experimental evidence to show that the ascent of micro-organisms from the urethra is the most common pathway leading to an UTI, especially organisms of enteric origin (i.e. Escherichia coli) [12]. This provides a logical explanation for the greater frequency of UTIs in women than in men and for the increased risk of infection following bladder catheterization or instrumentation [13]. Urinary tract infections cannot be diagnosed on clinical grounds alone. The hallmark of a UTI has been the presence of a single microorganism of $\geq 10^{5}$ colony forming units (cfus) per $\mathrm{ml}$ in a clean catch or midstream urine specimen, with lower numbers usually indicating contamination. Studies of the general population have suggested lower colony counts for reasons which include: an early stage of infection, recent use of antibiotics, cleaning the perineum with an antiseptic, frequency of urination, urinary tract obstruction, state of hydration (including the time of day the specimen was collected), urine $\mathrm{pH}$ or specific gravity, and orantibiotic therapy [14]. Pyuria is a term used to describe the appearance of increased numbers of ploymorphonuclear leucocyte in the urine and it is an evidence of acute inflammation. However, pyuria is not always associated with bacteriuria and its absence does not exclude infection [15].

The present work was an attempt to study the prevalence of organisms as well as to study the clino-pathological features of UTI from 85 patients of Global Baroda and Narhari Hospital, Baroda.

\section{Materials and Methodology}

Out of 85 patients, 80 were affected individuals and 5 were control patients which had symptoms similar to the 
affected individuals but a negative urine culture report. For instance, Pyuria without bacteriuria can be present with appendicitis, inflammatory bowel disease, and other extra renal disorders. The control group was set up so as to avoid such errors in selection of urinary tract patients. Out of the 80 affected patients, 44 were males and 36 were females.

The questionnaire was carried out to obtain brief history of these urinary tract infected patients about their current personal information regarding age, sex, address, occupation, past history of an infectious disease as well as age related complications and frequency of micturation, retention of urine, burning micturation, fever and chills. The samples were collected in pre-sterile, dry, wide-mouthed and leak proof universal plastic containers.

A clean-catch midstream specimen or suprapubic aspirate, in subjects who were unable to give the former, was collected in a sterile wide-mouth leak-proof container to hold about $50 \mathrm{ml}$ specimen. Using a calibrated loop method of a loop diameter of $4 \mathrm{~mm}, 10 \mu \mathrm{l}$ of the uncentrifuged specimen was transferred onto the agar plate and streak using the modified Mayo's technique without flaming the loop for isolation and incubated at $35^{\circ} \mathrm{C}-37^{\circ} \mathrm{C}$ for 24 hours. A specimen was considered positive for UTI if a single organism was cultured at a concentration of $>10^{5}$ colony-forming units $/ \mathrm{ml}$. Isolated colonies are identified in a systematic way: microscopic examination after Gram staining to differentiate between gram negative and gram positive bacteria. The Gram-positive and Gram-negative organisms were culture isolates which were further identified by using various biochemical reactions up to genus/species levels wherever applicable.

For Gram negative organisms: Sugar fermentation test, Indole test, Oxidase test, Methyl Red test, and urease test.

For Gram positive organisms: coagulase test and catalase test nutrient agar, blood agar and MacConkey’s medium are the frequently used growth media for urine cultures.

\section{Antibiotic Sensitivity Testing}

In the presence of any potential growth, antibiotic sensitivity testing was done by the modified Kirby-Bauer disc diffusion method according to the Clinical and Laboratory Standards Institute (CLSI) guidelines. The antibiotics tested were imepenem, meropenem, ciprofloxacin, ofloxacin, norfloxacin, amikacin, gentamicin, nitrofurantoin, and cotrimoxazole (Pathoteq Labs, India) was carried out using kit by Piramal healthcare \& Diasys diagnostic systems. Data of radiologic diagnostic methods such as ultrasound of abdomen (USG ABD) and urinary tract (USG KUB), MRI, CT scan along with various biochemical parameters to assess kidney function was also collected in patients who required such procedures.

\section{Results}

When the data from 80 positive samples was organized in different age groups as well as gender wise, it appeared that the overall prevalence of UTIs in males was about $10 \%$ higher than females in this study due to the high incidence of nosocomial UTI in the study group. The age group of 10 to 29 years showed 10\% (8 out of 80) risk for development of UTI. The age group of 30 - 49 showed a greater risk of developing UTI (23.75\%, 19). The age group which witnessed a dramatic increase in the incidence of UTI was 50 to 69 years with $41.25 \%$ (33 out of 80). Beyond the age of 70 years, the incidence of UTI declined to $22.5 \%$ at 70 to 89 years, which is a little higher (1.25\%) than the 30 to 49 years age group (Table 1 ).

There are many factors that predispose elderly persons to UTI, including chronic diseases, functional abnormalities, and specific medications. Also, women are more susceptible to UTI after menopause as there are low estrogen levels which can make it easier for bacteria to thrive in the vagina or urethra. Thus, estrogen has a protective effect in the urinary tract.

From the data of 80 patients, most frequently exhibited symptoms were anuria, burning micturation, dysuria, fever, haematuria, abdominal pain, pollakiuria and nausea. Burning micturation, abdominal pain and pollakiuria were the most common presenting symptoms in the present study. All the symptoms were more frequently present in uncomplicated UTI patients as compared to complicated UTI patients. This is due to the natural, gradual precipitation of an infection in urinary tract which allows for host defense factors to exert an immune response to some extent against the uropathogen, as observed in uncomplicated UTI (Table 2).

In 30 to 49 years age group, the incidence of diabetes mellitus (23.5\%; 19) was the most dominating risk factor followed by renal stones $(6.8 \%, 6)$, catheterization $(5.8 \%, 5)$, recent urogenital intervention $(4.2 \%, 4)$, and congenital anomalies $(3.2 \%, 3)$. Recurrent UTI $(2.5 \%, 2)$ and immunosuppression $(1.67 \%, 1)$ were relatively 
Table 1. Age-wise distribution of males and females with urinary tract infection.

\begin{tabular}{ccccc}
\hline Age group & Male & Percentage & Female & Percentage \\
\hline $10-19$ & 1 & 2.27 & 6 & 16.67 \\
$20-29$ & 2 & 4.54 & 1 & 2.78 \\
$30-39$ & 4 & 9.09 & 6 & 16.67 \\
$40-49$ & 6 & 13.63 & 3 & 8.33 \\
$50-59$ & 5 & 11.36 & 7 & 19.44 \\
$60-69$ & 16 & 36.36 & 5 & 13.89 \\
$70-79$ & 8 & 18.18 & 5 & 13.89 \\
$80-89$ & 2 & 4.54 & 3 & 8.33 \\
Total & 44 & 100.0 & 36 & 100.0 \\
\hline
\end{tabular}

Most of the cases were recorded in the elderly age group (50 - 79 years, 60.0\%). Adolescent (10 - 19 years) cases comprised of $8.75 \%$ of total cases.

Table 2. Frequency of risk factors in subjects with urinary tract infection.

\begin{tabular}{ccc}
\hline Risk factor & Freq & Percentage \\
\hline Catheterization & 25 & 32.9 \\
Diabetes mellitus & 31 & 40.8 \\
Immunosuppression & 4 & 5.3 \\
Recent urogenital intervention & 12 & 15.8 \\
Recurrent UTI & 11 & 14.5 \\
Renal stones & 28 & 36.8 \\
Congenital anomalies & 9 & 11.8 \\
\hline
\end{tabular}

Diabetes mellitus was the most common risk factor associated with UTI in the present study.

less common in this group. More dramatic results were gathered in the age group 50 to 69 years with diabetes mellitus $(41.6,33)$ predominating over all the other categories in all the age groups. Renal stones $(10 \%, 8)$, catheterization $(7.5 \%, 6)$, congenital anomalies $(4.2 \%, 4)$, and recent urogenital intervention $(3.2 \%, 3)$ and lowest incidences were of recurrent UTI $(2.5 \%, 2)$ and immunosuppression $(1.67 \%, 1)$. Persistence of diabetes mellitus $(7.5 \%, 6)$ declined greatly in 70 to 89 years age group and immunosuppression was completely absent.

Escherichia coli was the most frequently observed uropathogen in the present study with $38 \%$ incidence out of which it is observed more commonly seen in community-acquired UTI cases $(39.7 \%, 25)$ and nosocomial UTI cases (35\%). Klebsiella pneumoniae and Candida albicans hold second position in this study with 10\% each. K. pneumoniae is $9.3 \%$ \& $13.3 \%$, respectively and that of Candida albicans is $6.7 \%$ \& $1.7 \%$, respectively. Similar results have been noticed in all the prevailing organisms in the present study. Pseudomonas aeruginosa had an overall occurrence rate of $10 \%$. Staphylococcus aureus was present in $7.5 \%$ of all UTI cases. Proteus vulgaris had incidence of $6 \%$ of total UTI cases, followed by Proteus mirabilis with 3.75\% occurrence rate. Acinetobacter was least most frequent organism as observed in the present study with an overall occurrence of $1.25 \%$ (Table 3).

Aminoglycoside (Amikacin) and carbapenem (imipenem and meropenem) were the most frequently utilized antibiotics each with $16 \%$ sensitivity in nosocomial UTI and $41.3 \%$ sensitivity in community-acquired UTI. Second most frequently used aminoglycoside was gentamicin with sensitivity of $13.4 \%$ in nosocomial cases and $36 \%$ in community-acquired cases. Cyclic polypeptides held third position in being used as t treatment for UTI. Polymyxin B exhibited total sensitivity of $46.7 \%$ out of which $38.7 \%$ was seen in community-acquired UTI and only $8 \%$ was seen in nosocomial UTI. Colistin had a total sensitivity of $45.3 \%$ out of which $28 \%$ was seen in community-acquired UTI and $\mathbf{1 7 . 3 \%}$ was seen in nosocomial UTI. Fluoroquinolones (quinolones) were used less frequently as compared to aminoglycosides and carbapenems due to the increasing resistance of pathogens to this type of drugs. 
Table 3. Frequency of various pathogenic organisms in the study.

\begin{tabular}{ccccc}
\hline Organism & Nosocomial UTI & $\%$ & Community-acquired UTI & $\%$ \\
\hline Acinetobacter & - & - & 1 & 1.6 \\
Candida albicans & 3 & 17.7 & 5 & 7.9 \\
Enterococcus faecalis & 1 & 5.9 & 3 & 4.76 \\
Enterobacter & - & - & 2 & 3.17 \\
Escherichia coli & 6 & 35.3 & 25 & 39.7 \\
Gram negative bacilli & 1 & 5.9 & 3 & 9.76 \\
Klebsiella pneumoniae & 2 & 11.8 & 6 & 6.35 \\
Proteus vulgaris & 1 & 5.9 & 3 & 4.76 \\
Proteus mirabilis & - & - & 7 & 11.11 \\
Pseudomonas aeruginosa & 1 & 5.9 & 4 & 6.35 \\
Staphylococcus aureus & 2 & 11.8 & 63 & 100.0 \\
Total & 17 & 100.0 & & \\
\hline
\end{tabular}

E. coli is the most prevalent uropathogen in the present study. Acinetobacter being the rarest.

\section{Discussion}

The present study indicated that age was a major factor, as most of the cases were recorded in the elderly age group. These results were similar to those gained from other similar studies since Urinary tract infections are the most common infections in the elderly. There are many factors that predispose the elderly to UTI, including chronic disease, functional abnormalities and specific medications. Asymptomaticbacteuria is prevalent among this population, but it frequently resolves without the need of treatment and has no long-term sequelae. However, symptomatic UTI among the elderly requires stringent antimicrobial therapy. This age group is also more likely to develop serious complications as a result. In addition, women are more susceptible to UTI post-menopause as there are low estrogen levels which can make it easier for bacteria to thrive in the vagina or urethra. Thus, estrogen has a protective effect in the urinary tract. This study has acquired contradictory results as far as sex is concerned, as seen in normal scenarios, UTI is more common in women as compared to men, however, in this study, UTI is seen to be slightly more common in men than women. This may be due to the uncircumcised state of men, increased presence of indwelling catheter in men, low hygiene, multiple sexual partners etc. A more in depth study into the matter is required for a more clear judgement. Although UTI ranks among the most common infectious disease in developing countries, the quality of disease diagnosis and treatment are poor. Burning micturition was the most common symptom exhibited by the patients involved in the study but clinical presentation alone may play a small role, if any, in diagnosing UTI, reconfirming the fact that urine culture is essential for accurate diagnosis and designating a treatment regimen.

In the present study, diabetes mellitus was the most frequently observed risk factor which was present in complicated UTI patients belong to age group 30 to 89 years. Diabetes mellitus is associated with many complications and in the long run it has some major effects on the urinary system which makes diabetic patients more vulnerable to UTI and particularly to upper urinary tract infections. It has a long term effect on the incidence of UTI and it has been reported to be around four times higher in diabetics compared to non-diabetic patients. Diabetic nephropathy is one of the many factors that make these patients more susceptible to UTI than nondiabetics. Reduced immunity in diabetes also contributes to the increased risk for acquiring UTI. A few studies have suggested that hyperglycaemic urine promotes rapid bacterial growth and colonization. A range of infrequent presentations of complicated UTI such as pyelonephritis and pyelitis are commonly seen in patients with diabetes.

Catheterization was also a common risk factor in the present study leading to catheter-associated UTI in any age group. For either short- or long-term catheters, the infection rate is 5\% per day. Current recommendations are not to treat asymptomatic catheter-associated UTI as it leads to the emergence of drug-resistant organisms. To prevent infection, intermittent catheterization by either a nurse or by the patient is advised. In contrast to 
women, the occurrence of asymptomatic bacteriuria in men is rare until after 55 years of age, at which time the prevalence increases per decade and approaches the rate in elderly women. Prostatic hypertrophy and increased likelihood of instrumentation account for the bacteriuria in older men.

The uropathogen profile in our study is similar to other studies [16]. E. coil was the most frequently occurring uropathogen in both nosocomial as well as community-acquired UTI. However, other non-traditional uropathogens such as Candida albicans, Proteus vulgaris, and Staphylococcus aureus were more prevalent in nosocomial UTI cases. Klebsiella pneumoniae, Pseudomonas aeruginosa, Staphylococcus saprophyticus, Proteus mirabilis, Enterococcus faecalis are other common flora observed in urinary tract infections. E. coli are found in the urine of $80 \%-90 \%$ of patients with acute uncomplicated cystitis and acute uncomplicated pyelonephritis. Many patients with staghorn calculi harbour urea-splitting proteus organisms in their urine. Klebsiella, Pseudomonas and Enterobacter infections are commonly acquired in the hospital. The presence of Staphylococcus aureus often is a clue to concomitant Staphylococcal bacteremia, unless an underlying risk factor exists.

Analogous findings have been reported regarding the uropathogens' profile and their antibiotic sensitivity patterns in the presence of catheter-associated UTI [17]. Antibiotic sensitivity was lower in catheter-acquired UTIs as compared to community-acquired UTI. These results were similar to those observed in many other UTI related studies [18]. The antibiotic susceptibility pattern in the present study is similar to other studies [19]. Quinolones were the least active drug against uropathogens in the present study. The resistance rate for ciprofloxacin has been increasing over decades and this is the highest resistance rate reported to date. Akram et al. reported ciprofloxacinresistance rates ranging from $47 \%$ to $69 \%$ among the Gram-negative organisms in their study in India [20].

Although, quinolones were considered as one of the drugs of choice for the treatment of UTI, the increasing resistance rate necessitates a change in the empirical treatment against community-acquired UTI. Until recently, carbapenems were almost uniformly active against resistant Gram-negative organisms, but some strains have now developed very effective ways to deal with the carbapenems. There are various mechanisms by which these organisms achieve such feat, by producing beta lactamases which destroy the antibiotics, by blocking the entry of these antibiotics, or by efflux pumps which actively pump out these antibiotics [21].

Kader et al. reported 8.9\% ESBL-positive cases in a hospital-based study in Saudi Arabia [22]. Bean et al. reported a community-based ESBL prevalence to be 5.7\% in London. In the present study, $51.2 \%$ of the isolates were ESBL-positive uropathogens. Previous studies in India have reported an ESBL positivity rate between 26.9\% and $48.3 \%$ [23]. ESBL producers do not respond to the usually prescribed empirical therapy. Also there is an increased risk of associated morbidity and mortality, and cost of therapy when these patients are put on the standard empirical therapy. Presently, alternative antimicrobial therapy to treat ESBL-positive UTI on outpatient basis is limited. Carbapenems are the most effective in this situation but need to be administered intra-venously oramuscularly. The experimental use of fosfomycin in treating ESBL-positive UTI has also shown promising results in the recent past. All this and the high rate of ESBL positivity in the present study warrant a change in the empirical therapy for UTI to prevent the complications.

The uropathogens showed highest sensitivity to carbapenems. The next best alternatives to treat complicated and drug-resistant cases were aminoglycosides. Though the bacterial spectrum causing community-acquired UTI remained the same over time, the antibiotic susceptibility has changed. With limited options and all the above-mentioned growing concerns, it would not be late where we will find ourselves in epidemics with multidrug-resistant organisms. We now have to find alternative and economical options to fend off an otherwise catastrophe.

\section{Conclusions}

The present study was conducted in order to evaluate the clino-pathological features of Urinary Tract Infection as observed in patients of Global Baroda Hospital and Narhari Hospital, Baroda. A questionnaire was prepared in accordance which interviewed the UTI patients about their medical histories and lifestyles. The data obtained were analysed to study the UTI risk factors such as age, sex, diabetes mellitus, catheterization, renal stones, immunosuppression, congenital anomalies and recurrent UTI. The characteristics of Urinary Tract Infection in Baroda as per our study are as follows:

1) Increased age is associated with an increase in risk of acquiring urinary tract infection.

2) Males are prone to urinary tract infection than females (55 males and 45 females). 
3) Urinary tract infections are more commonly suspected in post-menopausal women as compared to teenage women as well as women in their early 20 s.

4) The peak period of urinary tract infection is attained between 50 to 69 years.

5) Clinical presentation alone plays a minor role in establishing diagnosis in UTI; however its role is maximized when accompanied by a urine culture.

6) Diabetes, renal calculi and urogenital instrumentation were the major risk factors for UTI.

7) E. coli is still the most widely prevalent organism causing UTI in the community as well as nosocomial UTI although antibiotic sensitivity spectrum is different for both the pathogenic strains.

8) Carbapenems were the most sensitive drugs and widely used on patients of this study group.

9) Quinolones were the least sensitive drugs and were used in rare cases throughout the present study due to the increasing rate of its resistance.

10) Complications related to UTI as seen in the present study included kidney failure (8.75\%), development of sepsis as a result of bacteremia (11.25\%), multi-organ failure and death (6.25\%).

The current understanding on the risk factors of urinary tract infection emphasizes that age, gender and diabetes mellitus are unavoidable risk factors, but the modifiable risk factors such as renal stone can be prevented by removal of the stone immediately, followed by a 3-day antimicrobial therapy to eradicate presence of any uropathogen aggregated with the renal calculi. Also urine culture should be necessitated in patients suspected with UTI in order to establish proper treatment and avoid the need to begin empirical therapy which may be a contributor to the increasing number of ESBL species.

To summarize, the current scenario of urinary tract infection reveals alarmingly high rate of resistant ESBL species which should draw our attention; the resistance pattern, though not that different from the rest of the world, is ever increasing due to uncontrolled abuse of the available antibiotics. A strong decision has to be established regarding the antibiotic policies for UTI and stringent measures have to be taken to ensure the effectiveness of the same. Failing to do so, the time is not far where we would have to stand helplessly against these uropathogens.

\section{Acknowledgements}

We acknowledge assistance and cooperation by the medical staff of Narhari Hospital and Global Baroda Hospital, Vadodara.

\section{Limitations}

This study was conducted with the aim to uncover and bring to light several pathological aspects that correlate with the clinical representation of Urinary tract infections which are commonly occurring in rural areas of developing countries such as India. Due to the time constraint as well as financial limitations further research into the design of an empirical therapeutic dose to treat the urinary infections and also to create awareness about the role of hygiene in the same could not be followed through. Though tests and methods mentioned above were carried out accurately and with full dedication to give trustworthy results for additional analysis on the subject.

\section{Disclosures}

No conflicts of interest, financial or otherwise, are declared by the author(s).

\section{References}

[1] Gonzalez, C.M. and Schaeffer, A.J. (1999) Treatment of Urinary Tract Infection: What's Old, What's New, and What Works. World Journal of Urology, 17, 372-382. http://dx.doi.org/10.1007/s003450050163

[2] Kunin, C.M. (1997) Detection, Prevention and Management of UTIs. 5th Edition, Lea \& Febiger, Philadelphia.

[3] Mobley, H.L.T., Island, M.D. and Massad, G. (1994) Virulence Determinants of Uropathogenic Escherichia coli and Proteus mirabilis. Kidney International, 46, S129-S136.

[4] Stamm, W.E. and Hooton, T.M. (1993) Management of Urinary Tract Infections in Adults. New England Journal of Medicine, 329, 1328-1334. http://dx.doi.org/10.1056/NEJM199310283291808

[5] Percival, A., Birumfitt, W. and Delouvois, J. (1964) Serum Antibody Levels as an Indication of Clinically Unapparent Pyelonephritis. Lancet, 2, 1027-1033. http://dx.doi.org/10.1016/S0140-6736(64)90988-2 
[6] Naber, K.G. and Weidner, W. (1999) Prostatitits, Epididymitis and Orchitis. In: Armstrong, D. and Cohen, J., Eds., Infectious Diseases, Mosby, London, Chapter 58.

[7] Gulati, S., Kher, V., Gupta, A., Arora, P., Rai, P.K. and Sharma, R.K. (1995) Spectrum of Infections in Indian Children with Nephrotic Syndrome. Pediatric Nephrology, 9, 431-434. http://dx.doi.org/10.1007/BF00866719

[8] Kunin, C.M. (1994) Urinary Tract Infections in Females. Clinical Infectious Diseases, 18, 1-12. http://dx.doi.org/10.1093/clinids/18.1.1

[9] Meyrier, A. (1996) Diagnosis and Management of Renal Infections. Current Opinion in Nephrology and Hypertension, 5, 151-157. http://dx.doi.org/10.1097/00041552-199603000-00009

[10] Bailey, R.R. (1994) Single-Dose/Short-Term Therapy in Children and in Pregnant Women. Infection, 22, S47-S48. http://dx.doi.org/10.1007/bf01716044

[11] Emori, T.G. and Gaynes, R.P. (1993) An Overview of Nosocomial Infections, including the Role of the Microbiology Laboratory. Clinical Microbiology Reviews, 6, 428-442.

[12] Roberts, J.A. (1991) Etiology and Pathophysiology of Pyelonephritis. American Journal of Kidney Diseases, 17, 1-9. http://dx.doi.org/10.1016/S0272-6386(12)80242-3

[13] Raz, R. and Stamm, W.E. (1993) A Controlled Trial of Intravaginal Estriol in Postmenopausal Women with Recurrent Urinary Tract Infections. New England Journal of Medicine, 329, 753-756. http://dx.doi.org/10.1056/NEJM199309093291102

[14] Wullt, B., Bergsten, G. and Fischer, H. (2004) Application of Laboratory Research in UTI. European Urology EAU Update Series, 2, 116-124. http://dx.doi.org/10.1016/j.euus.2004.06.004

[15] Kass, E.H. (1956) Asymptomatic Infections of the Urinary Tract. Transactions of the Association of American Physicians, 69, 56-64.

[16] Dumanski, A.J., Hedelin, H., Edin-Liljergen, A., Beauchemin, D. and McLean, R.J. (1994) Unique Ability of the Proteus Mirabilis Capsule to Enhance Mineral Growth in Infectious Urinary Calculi. Infection and Immunity, 62, 29983003.

[17] Kahlmeter, G. (2000) The ECOSENS Project: A Prospective, Multinational, Multicentre Epidemiological Survey of the Prevalence and Antimicrobial Susceptibility of Urinary Tract Pathogens-Interim Report. Journal of Antimicrobial Chemotherapy, 46, 15-22. http://dx.doi.org/10.1093/jac/46.suppl_1.15

[18] Gupta, K. (2003) Emerging Antibiotic Resistance in Urinary Tract Pathogens. Infectious Disease Clinics of North America, 17, 243-259. http://dx.doi.org/10.1016/S0891-5520(03)00006-0

[19] Engel, J.D. and Schaeffer, A.J. (1998) Evaluation of and Antimicrobial Therapy for Recurrent Urinary Tract Infections in Women. Urologic Clinics of North America, 25, 685-701. http://dx.doi.org/10.1016/S0094-0143(05)70057-4

[20] Akram, M., Shahid, M. and Khan, A.U. (2007) Etiology and Antibiotic Resistance Patterns of Community-Acquired Urinary Tract Infections in JNMC Hospital Aligarh, India. Annals of Clinical Microbiology and Antimicrobials, 6, 4. http://dx.doi.org/10.1186/1476-0711-6-4

[21] Hotchkiss, R.S. and Karl, I.E. (2003) The Pathophysiology and Treatment of Sepsis. New England Journal of Medicine, 348, 138-150. http://dx.doi.org/10.1056/NEJMra021333

[22] Kader, A.A. and Angamuthu, K. (2005) Extended-Spectrum Beta-Lactamases in Urinary Isolates of Escherichia coli, Klebsiella pneumoniae and Other Gram-Negative Bacteria in a Hospital in Eastern Province, Saudi Arabia. Saudi Medical Journal, 26, 956-959.

[23] Bean, D.C., Krahe, D. and Wareham, D.W. (2008) Antimicrobial Resistance in Community and Nosocomial Escherichia coli Urinary Tract Isolates, London 2005-2006. Annals of Clinical Microbiology and Antimicrobials, 7, 13. http://dx.doi.org/10.1186/1476-0711-7-13 